\title{
Photonic Properties of Strongly Correlated Colloidal Liquids
}

\author{
L. F. Rojas-Ochoa, ${ }^{1}$ J. M. Mendez-Alcaraz ${ }^{2}$ J. J. Sáenz, ${ }^{3}$ P. Schurtenberger, ${ }^{1}$ and F. Scheffold ${ }^{1, *}$ \\ ${ }^{1}$ Department of Physics, University of Fribourg, 1700 Fribourg, Switzerland \\ ${ }^{2}$ Departamento de Física, CINVESTAV-IPN, Avenida IPN 2508, Colonia San Pedro Zacatenco, 07300 México City, México \\ ${ }^{3}$ Departamento de Física de la Materia Condensada, and Instituto "Nicolás Cabrera," Universidad Autónoma de Madrid, \\ E-28049 Madrid, Spain
}

(Received 24 September 2003; published 13 August 2004)

\begin{abstract}
The optical and structural properties of dense colloidal suspensions in the presence of long-range electrostatic repulsion are determined from both light and small-angle neutron scattering experiments. Short-range structural order induces an enhancement of the scattering strength while at the same time the total transmission shows strong wavelength dependence, reminiscent of a photonic crystal. Interestingly, the interplay between diffusive scattering and local order leads to negative values of the scattering anisotropy parameter. The tunable optical properties of these liquids furthermore suggest potential applications such as transparency switches or filters.
\end{abstract}

DOI: 10.1103/PhysRevLett.93.073903

PACS numbers: 42.25.Bs, 42.70.Qs, 82.70.Dd

When light is incident on a nonabsorbing material, its further propagation is strongly influenced by the microscopic structure of the material itself. For a bulk homogeneous medium, light is refracted according to Snell's law. Local variations in the dielectric properties lead to isolated scattering events that disperse the light beam. The scatterer density and cross section define the scattering mean free path $l$. As the number of scattering events increases, the transport of light becomes diffusive and the material appears turbid or "white" [1,2]. The relevant scattering length for diffusive light transport is the transport mean free path $l^{*}$. Both quantities are connected by the scattering anisotropy parameter $g$ defined as the average of the cosine of the scattering angle $g=\langle\cos \theta\rangle$, $l / l^{*}=1-g$. Our current understanding of the diffusive transport is based on the knowledge of these key quantities. In the absence of positional correlations, $l^{*}$ is usually equal to or larger than $l$ [2-5]. For instance, Mie particles (or human tissue [1]) scatter strongly in the forward direction (small scattering angles $\theta$ ) and hence $g \simeq 1$ while for Rayleigh scatterers $g \simeq 0$. Here we show that these common properties of diffusive transport can be manipulated by tuning the interaction between scatterers. By the appropriate control of the Coulomb repulsion between highly charged particles in suspension, we are now able to access the whole possible interval of $g$ values (from forward scattering $g \rightarrow 1$ to the unusual case of strong backscattering $g \rightarrow-1$ ).

When mesoscopic variations of the dielectric constant can be neatly controlled over macroscopic distances, totally new, so-called photonic properties may appear [6]. At the core of the design of new photonic materials lies the intelligent way structures are assembled on length scales comparable to the wavelength of light. There are two main promising concepts to achieve lossless guidance and manipulation of light based on seemingly opposite principles: order or disorder. Photonic band gap materials are based on periodic structures predicted to inhibit light propagation completely [6]. In the case of disorder, light cannot propagate in the material due to recurrent interference called strong Anderson localization [7].

Tailoring microstructures with an appropriate degree of order and disorder should result in a new class of materials with unusual transport properties for light [8]. Here we have chosen a bottom-up self-assembly approach to control local order by working with colloidal liquids rather than the solid phases usually studied. As we show, short-range-order induced Bragg backscattering resonances lead to strong wavelength dependence of the optical properties of colloidal liquids. By tuning the interaction potential between the particles we are able to control the degree of order or disorder and thus explore photonic properties in a completely new regime.

In our experimental approach we make use of the peculiar properties of highly charged particles in suspension: Coulomb repulsion leads to pronounced positional correlations such that the particles stay, on average, at the maximum possible distance $d$ [9]. One key element of our study is the preparation of these dense colloidal suspensions in a regime where the electrostatic interaction potential is strong and long ranged, but crystallization is suppressed. Such systems are not readily available since in typical salt-free aqueous suspensions crystallization already occurs at very low volume fractions [9] $\Phi \leq 10^{-3}$. However, the onset of crystallization can be nicely tuned when a mixture of water and ethanol is used as a solvent [10]. Our sample consists of negatively charged polystyrene particles with a mean radius of $R=$ $57 \pm 2.5 \mathrm{~nm}$ and intrinsic polydispersity of ca. $9 \%$ (from dynamic light scattering). We selectively choose the solvent ionic strength, and thereby tune the interaction between particles from charged spheres to hard spheres. Samples have been prepared by reverse osmosis of the stock solution (from Interfacial Dynamics Corporation) 
in a mixture of ethanol and water at the desired ratio ( $75 \%$ and $25 \%$ in volume). The index of refraction of the particles and solvent are $n_{p}=1.59, n_{s}=1.365$. From the dialyzed sample $\Phi \approx 0.2$ we prepared a complete set of concentrations in the range of interest, $\Phi=0.02-0.16$. In order to fully deionize the samples, they were kept at least 10 days in contact with equal volumes of mixed bed ion exchanger resin (Dowex) prior to measurement. After the optical characterization some of the more concentrated samples were transferred to new cells and approximately $1 \%$ electrolyte (KCL in water) was added to screen electrostatic repulsions (final ionic strength approximately $5 \mathrm{mM}$ ). This procedure essentially leads to a hard-sphere interaction potential (screening length $\kappa^{-1} \approx 3 \mathrm{~nm}$ ).

We have determined the suspension scattering strength, or optical density $1 / l^{*}$, by measurements of the total transmission (Laser wavelength $\lambda_{L}=532 \mathrm{~nm}$, in solvent $\lambda=\lambda_{L} / n_{s}=390 \mathrm{~nm}$ ) following the approach of Kaplan et al. [11] using rectangular quartz cells of different thicknesses $(L=2-5 \mathrm{~mm})$ and assuring $L / l^{*} \geq$ 10 (Fig. 1).

For a noncrystalline, nonabsorbing medium the coefficient for total transmission $T$ is related to the mean free path through Ohm's law,

$$
T \propto \frac{l^{*}}{L} ; \quad L \gg l^{*},
$$
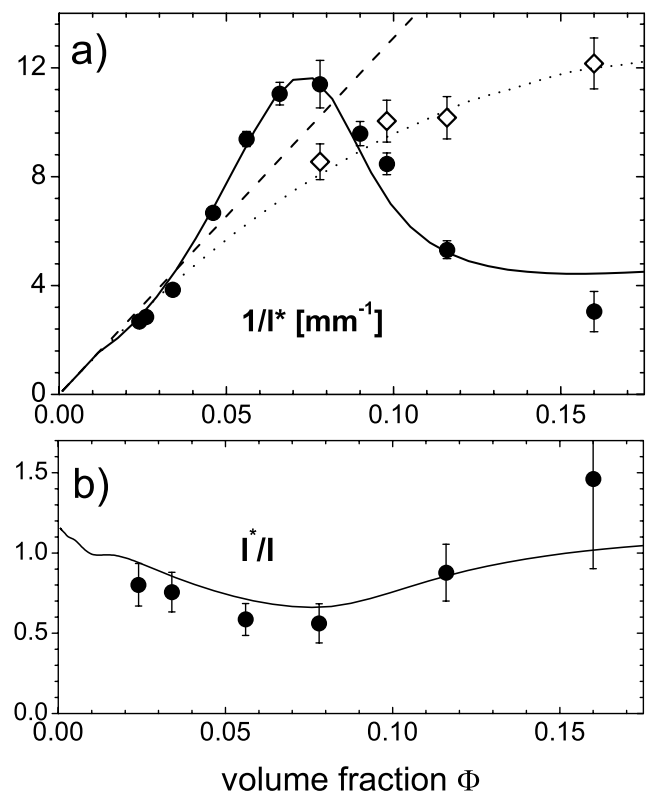

FIG. 1. (a) Optical density $1 / l^{*}$ of polystyrene particle suspensions (mean radius $57 \mathrm{~nm}$ ) at different concentrations (volume fraction $\Phi$ ) for $\lambda=\lambda_{L} / n_{s}=390 \mathrm{~nm}$ (full symbols: charged spheres; open symbols: hard spheres). Solid line: Calculation for charged spheres with an effective charge per particle $Z_{\text {eff }}=300 e^{-}$. Dotted line: theoretical calculations for hard-sphere interactions. Dashed line: no interactions $[S(q) \equiv 1]$. (b) Ratio of the transport mean free path and the scattering mean free path (solid circles). Solid line: theoretical calculation based on Eq. (3). in full analogy to the case of disordered electronic conductors. For uncorrelated scatterers (dilute limit) the optical density $1 / l^{*}$ increases linearly with the particle volume fraction: $1 / l^{*} \propto \Phi$. As the concentration increases, excluded volume interactions induce positional correlations that usually lead to much increased $l^{*}$ values [2-4]: the most disordered sample is expected to scatter most efficiently, and furthermore for $\Phi \rightarrow 1$ (no scattering contrast) $1 / l^{*}$ vanishes. For the simple case of hardspheres-like systems this behavior has indeed been confirmed for different particle sizes and bimodal mixtures [3-5]. Note that these positional correlations are also responsible for the relatively long electron mean free paths measured in disordered liquid metals $[12,13]$.

Our experimental results, however, draw a completely different picture: Over a wide range of concentrations, the charged systems are found optically more dense compared to the hard-sphere systems (and even compared to the noncorrelated limit, $1 / l^{*} \propto \Phi$ in strong contrast to previous results $[3,4])$. To understand this unusual enhancement of scattering strength we have to look in more detail at how the local order influences the transport of light. In the weak scattering limit $\left(k_{0} l^{*} \gg 1\right)$ a quantitative relation between $l^{*}$ and the (liquid) structure factor $S(q)$ is given [3] via the number density $\rho$ and the transport cross section $\sigma_{t r}$ :

$$
\frac{1}{l^{*}}=\rho \sigma_{t r}=\frac{\pi}{k_{0}^{6}} \int_{0}^{2 k_{0}} \rho F(q) S(q) q^{3} d q,
$$

where $q=2 k_{0} \sin \Theta / 2, k_{0}=2 \pi n_{s} / \lambda_{L}$ for a scattering angle in the range $\Theta=0-180^{\circ}$. The form factor is given by the differential cross section of an isolated particle $F(q)=k_{0}^{2}\left(d \sigma_{s c} / d \Omega\right)$. This is the same relation entering in the formal theory of electrical resistance [12]. For colloids at low ionic strength (and for electrons in a disordered Fermi liquid [14]) screening of Coulomb repulsions is inhibited, which in turn leads to an extended correlation hole in the radial distribution function. The preferred interparticle distance $d \cong \rho^{-1 / 3}$ is given by the particle number density, and a pronounced peak in $S(q)$ is found at $q_{\max } \approx 2 \pi / d$. Experimental results for $S(q)$ were obtained from small-angle neutron scattering (SANS) as shown in Fig. 2. Details of the experiment and the data treatment are given in Ref. [5].

Numerically we obtain the static structure factor $S(q)$ by solving the Ornstein-Zernike equation through the hypernetted chain closure relation for the charged-spheres case (excess salt concentration $2 \times 10^{-7} \mathrm{M}$ ) and the Percus-Yevick closure relation for the hard-spheres case (polydisperse versions) $[15,16]$. Particle size and polydispersity, as obtained from dynamic light scattering, were used as input to the theoretical model. In the range studied we find the best agreement with the $I(q)$ data (using the differential particle cross section $d \sigma_{s c}$ / $d \Omega$ for neutron scattering [5]) for an effective charge per particle in the range $275 e^{-}<Z^{\text {eff }}<325 e^{-}$. We note 
that for $\Phi>0.074$ a value of $S\left(q_{\max }\right)>3$ was found, indicating a supercooled liquid or glassy state [15]. A more detailed analysis of the structural and dynamic properties will be presented elsewhere.

For the observed minimum in the total transmission at $\Phi \simeq 0.074$ [Fig. 1(a)] we find $2 d=4 \pi / q_{\max }=389 \mathrm{~nm}$ matching exactly the Bragg condition, $d=\lambda / 2$, at normal incidence $(\lambda=390 \mathrm{~nm})$. This suggests Bragg backscattering as the origin of the unexpected strong concentration dependence of $1 / l^{*}$ shown in Fig. 1 [17].

In this context we should stress that the transport properties rely not only on the interplay between order and disorder but also on the scattering properties of individual particles. Mie particles $\left(k_{0} R>1\right)$ possess an increased scattering cross section, but at the same time strong forward scattering might inhibit the backscattering effects described before. Resonant backscattering is expected for $\rho^{-1 / 3} \cong d \approx \lambda / 2$; hence, for a given particle size the resonant volume fraction is $\Phi_{\text {res }} \approx$ $4 \pi \rho R^{3} / 3=4 / 3 \pi^{2} \times\left(k_{0} R\right)^{3}$. This result reflects the simple fact (since $\Phi<1$ ) that the particle size sets a lower bound for the interparticle distance, $d \geq 2 R$; hence, for $k_{0} R>\pi / 2$ resonant behavior is precluded. However, for $k_{0} R \geq 1$ where the single particle cross section (per particle volume) is maximal and forward scattering is still moderate, resonant scattering is very possible for $\Phi \approx 0.1-0.65$.

Close to a Bragg resonance the scattering cross section is expected to be anisotropic. In our systems this anisotropy should be reflected in a strong (and unusual) difference between transport mean free path $l^{*}$ and scattering mean free path $l$ : Both quantities, $l$ and $l^{*}$, are connected

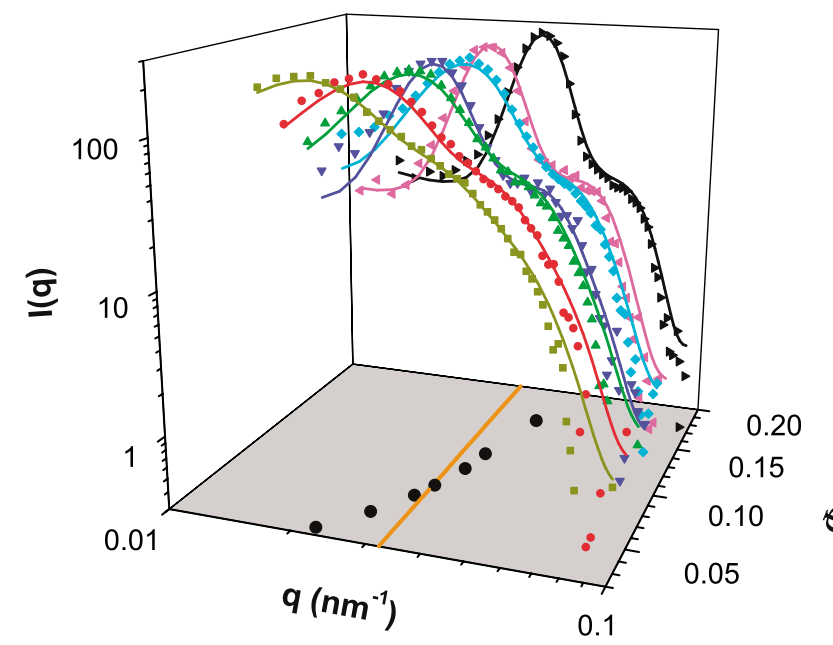

FIG. 2 (color online). Liquid structure for charged spheres: $q$-dependent neutron scattering intensity $I(q)$ for different particle concentrations $\Phi$. The position of the scattering peak (solid circles) scales with the typical interparticle distance, $q_{\max }=2 \pi / d \sim \Phi^{1 / 3}$. Solid lines: Numerical fits to the data with an effective charge ranging from $Z_{\text {eff }}=275 e^{-}$to $325 e^{-}$. A maximum in the optical density, Fig. 1, is observed when $q_{\max }$ crosses the orange line at $2 k_{0}=0.0322 \mathrm{~nm}^{-1}$. by the scattering anisotropy parameter $g$ :

$$
\frac{l^{*}}{l}=\frac{1}{1-g}=2 k_{0}^{2} \frac{\int_{0}^{2 k_{0}} F(q) S(q) q d q}{\int_{0}^{2 k_{0}} F(q) S(q) q^{3} d q} .
$$

We have determined experimentally the scattering mean free path $l$ by line-of-sight transmission measurements (unscattered beam attenuation) using a focused laser beam and reduced optical paths between 50 and $1000 \mu \mathrm{m}$. The results are shown in Fig. 1(b). For $\Phi<$ 0.1 we find $l^{*}$ significantly smaller than $l$. At the same time the transmission coefficient is indeed reduced. Notice that the measured ratio at the Bragg condition is close to $\frac{1}{2}$. To our knowledge this is the first clear evidence for a negative anisotropy parameter [18]. This finding has immediate consequences on many aspects of multiple wave scattering since it is now possible to study $g$-dependent properties (such as the polarization memory of multiply scattered light [19]) over the full range of possible $g$ values.

In Fig. 3 we show the transmission wavelength dependence for a concentration $\Phi=0.097$. The data were obtained using a standard UV-VIS spectrometer (PerkinElmer Lambda 19). Again, at the resonance wavelength, we observe a pronounced dip in the normalized transmission (inset of Fig. 3). To describe the data on absolute scales and over the full wavelength range Eq. (1) has been extended (as described in Ref. [20]), taking into account low order scattering and surface reflectivity. As shown in Fig. 3 excellent agreement is obtained with $l$ and $l^{*}$ from Eqs. (2) and (3) and an effective reflection coefficient 0.45 .

From the discussion above, we can conclude that the strong wavelength dependence of our photonic liquids can

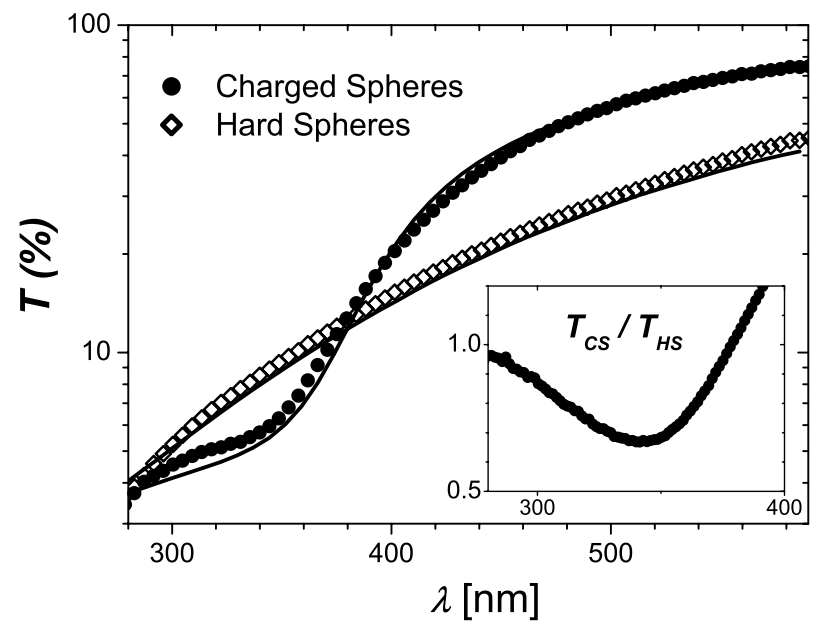

FIG. 3. Transmission wavelength dependence. Experimental values for charged spheres (solid symbols) and hard spheres (open symbols), $\Phi=0.097$. Cell thickness: $L=2 \mathrm{~mm}$. Solid lines: Theoretical calculations taking into account low order scattering and surface reflectivity. Inset: The transmission ratio shows a minimum at $\lambda \approx 341 \mathrm{~nm}$ [Bragg condition $\lambda=2 d=$ $\left.4 \pi / q_{\max }=356 \mathrm{~nm}\right]$. 
be associated with a (short-range order induced) Bragg matching resonance. Interestingly, it has been recently predicted [14] that for disordered electronic conductors with short-range correlations, this matching condition (for the electron wavelength at the Fermi level) would lead to a pseudogap in the electron density of states. The increased scattering strength of photonic liquids together with a reduced photon density of states could then open an alternative pathway to strong localization of light in disordered structures [21], closer to John's original proposal [22] than other previous attempts [23].

Finally, we point out that the unusual properties of photonic liquids could lead to interesting applications. Titanium dioxide nanoparticle based sunscreen lotions, for example, require efficient blocking of UV light while retaining a high transparency for visible wavelengths. As we have shown in Fig. 3, photonic liquids fulfill this requirement significantly better than random particle assemblies. Photonic liquids could also be used as tunable optical filters and switches, for example, in windows that change from opaque to clear, provided particle interactions can be controlled externally. Recently it has been shown that under well defined conditions an external electric field can play the role of an effective temperature and thus modulate the interaction potential in a controlled way [24]. Although our systems are different, the strong wavelength dependence of our photonic liquids together with a similar field effect suggests intriguing and exciting applications in manipulating the propagation of light.

We thank C. Soukoulis for discussions and H. Kroha for pointing out the analogy to disordered alloys. SANS experiments were performed at the Swiss Spallation Neutron Source, Paul Scherrer Institute, Villingen $(\mathrm{CH})$. We thank A. Stradner and J. Kohlbrecher for their help with the SANS measurements and the data treatment. J. J. S. thanks R. Carminati and J. J. Greffet for their hospitality during his sabbatical at Ecole Centrale Paris (supported by the Spanish MECyD, Ref. No. PR20020161). Financial support by the Swiss National Science Foundation is gratefully acknowledged.

*Corresponding author.

Electronic address: Frank.Scheffold@unifr.ch

Electronic address: www.unifr.ch/physics/mm/

[1] P. Sebbah, Waves and Imaging through Complex Media (Kluwer Academic Publishers, Dordrecht, Boston, 2001).

[2] G. Maret, Recent Experiments on Multiple Scattering and Localization of Light (North-Holland, Amsterdam, 1995).

[3] S. Fraden and G. Maret, Phys. Rev. Lett. 65, 512 (1990).

[4] P. D. Kaplan, A. G. Yodh, and D. J. Pine, Phys. Rev. Lett. 68, 393 (1992).

[5] L. F. Rojas-Ochoa et al., Phys. Rev. E 65, 051403 (2002).
[6] E. Yablonovitch, Phys. Rev. Lett. 58, 2059 (1987); J. D. Joannopoulos, R. D. Meade, and J. N. Winn, Photonic Crystals: Molding the Flow of Light (Princeton University Press, Princeton, NJ, 1995); C. M. Soukoulis, Photonic Crystals and Light Localization in the 21st Century (Kluwer Academic Publishers published in cooperation with NATO Scientific Affairs Division, Dordrecht, Boston, 2001).

[7] P.W. Anderson, Philos. Mag. B 52, 505 (1985); S. John, Phys. Rev. Lett. 58, 2486 (1987).

[8] J. Chongjun et al., Phys. Rev. B 63, 195107 (2001); J. Ballato et al., Appl. Phys. Lett. 75, 1497 (1999).

[9] W. B. Russel, D. A. Saville, and W. R. Schowalter, Colloidal Dispersions (Cambridge University Press, Cambridge, NY, 1989).

[10] L. F. Rojas et al., Europhys. Lett. 60, 802 (2002).

[11] P. D. Kaplan et al., Appl. Opt. 32, 3828 (1993).

[12] J. M. Ziman, Philos. Mag. B 6, 1013 (1961).

[13] N.W. Ashcroft and J. Lekner, Phys. Rev. 145, 83 (1966).

[14] J. Kroha, A. Huck, and T. Kopp, Phys. Rev. Lett. 75, 4278 (1995).

[15] J. P. Hansen et al., in Liquids, Freezing and Glass Transition, Proceedings of the Les Houches Summer School, Session LI (North-Holland, Amsterdam, New York, 1991).

[16] B. D’Aguanno and R. Klein, Phys. Rev. A 46, 7652 (1992).

[17] Though, to our knowledge, it has not been observed before, the phenomena reported here are of rather general nature, closely related to the physics of Bragg diffraction rings in disordered crystals. Whenever the position of the structure factor peak matches the resonance condition, this effect should be present in strongly scattering media.

[18] Negative $g$ values have been proposed to indicate the proximity of strong Anderson localization [J. Gomez Rivas et al., Phys. Rev. E 63, 046613 (2001)]. We note that the actual finding of negative $g$ values in this previous work is ambiguous due to the large error bars. Here we describe a simple physical mechanism that leads to negative $g$ values (resonant Bragg backscattering).

[19] L. F. Rojas et al., cond-mat/0402391.

[20] P. A. Lemieux, M. U. Vera, and D. J. Durian, Phys. Rev. E 57, 4498 (1998).

[21] For disordered crystal structures John suggested [22] that the fluctuations of the wave vector around the Bragg plane become relevant, rather than the free wave number, thus renormalizing the Ioffe-Regel criterion. We speculate that for photonic liquids this width should roughly correspond to the width of the first peak in the structure factor and the Ioffe-Regel condition could thus be met much more easily compared to completely random configurations.

[22] S. John and R. Rangarajan, Phys. Rev. B 38, 10101 (1988).

[23] D. S. Wiersma et al., Nature (London) 390, 671 (1997); F. Scheffold et al., Nature (London) 398, 206 (1999); A. A. Chabanov, M. Stoytchev, and A. Z. Genack, Nature (London) 404, 850 (2000).

[24] A. Yethiraj and A. van Blaaderen, Nature (London) 421, 513 (2003) 